\section{Rate of Decay of Polonium in Different Points of the U.S.S.R.}

THE half-period of a radioactive element characterises the rapidity with which it decays. If the classical theory of the spontaneously exploding atom be aceepted, this rate should be the same at any point of the earth's surface.

In order to verify this assumption, measurements of the half-period of polonium have been made during the past two years. Polonium was chosen for this purpose, as the most convenient radioactive sub. stance for observations of this kind, because it is easily obtained in a pure state, its half-period can be directly observed $(136 \cdot 5 \pm 0 \cdot 3$ days $)$, and it is also the last radioactive member of the uranium series.

In these experiments polonium was deposited electrolytically on accurately polished gilt brass discs of $75 \mathrm{~mm}$. diameter to avoid the possibility of the oxidation of metallic surface. Discs having small rims were supplied with round covers which safely protected the active layer from mechanical effects. The process of carrying out the experiments was as follows: The dises were carefully measured by means of a compensating electrometric set which allowed their activity to be determined through the magnitude of the ionisation current with an accuracy of 0.2 per cent. The set itself was verified by means of a uranium standard. Just after this measurement the active dises were packed, sealed up and sent by post to a number of places, where they were kept according to instructions in the Local Weights and Measures Offices, which are under the management of the Central Chamber of Weights and Measures.

After an interval of about five months, packets containing the discs were returned to Leningrad and were immediately measured for the second time. The half-period was calculated according to the formula expressing the rate of decay : $I_{t}=I_{0} e^{-\lambda t}$, and to the equation $T=\log 0.5 \times I / \lambda$ where $I_{0}$ is the initial activity before sending to the points; $I_{t}$, the activity after the receipt in Leningrad; $t$, the time between two measurements; $\lambda$, the radioactive constant; and $e$ the base of natural logarithms.

The determinations of the half-periods were made at eighteen points corresponding to eighteen towns, namely, Murmansk (1), Archangelsk (2), Leningrad (3), Vologda (4), Kazan (5), Moscow (6), Samara (7), Kursk (8), Saratov (9), Charkow (10), Rostow/Don (11), Odessa (12), Astrachan (13), Krasnodar (14), Wladikaukas (15), Tiflis (16), Baku (17), Erivan (18).

The most northern point was Murmansk $\left(68^{\circ} 59^{\prime}\right.$ N.) and the most southern one was Erivan in Caucasus $\left(40^{\circ} 11^{\prime}\right.$ N.).

All the points were distributed through a distance of $3000 \mathrm{~km}$. along the meridian. The results obtained show that the rate of decay of polonium is far from being equal in all points. The value of the period changed from $125 \cdot 6$ days (Tiflis) to $181 \cdot 6$ days (Krasnodar). A significant reduction for Astrachan gave the value $127 \cdot 8$ days. The average least square error of the observations did not surpass 0.7 per cent.

From the results obtained we reach the conclusion that, taking into consideration the absence of influence of the metal, which could only reduce the value of the half-period, local conditions had an influence upon the rapidity of radioactive decay.

To verify our assumption, the determinations of the half-period were repeated for all eighteen discs after they had lain about five months in Leningrad. The values of the period thus found varied from $137 \cdot 2$ to 139.5 days, which is not outside the limits of observation errors. On the accompanying diagram (Fig. 1) curve $A$ shows the values of the half-period in different places, and curve $B$ the values of the half-period in Leningrad.

The experiments described are preliminary only, and the investigation will be undertaken on a larger scale with the view of determining without any doubt the influence of local conditions upon the rate of decay of radioactive elements.

This phenomenon can be easily explained, if we admit Perrin's theory assumption of the existence of an external source of radiant energy which produces the radioactive decay of atoms. If we admit the existence of this source in the centre of the earth,

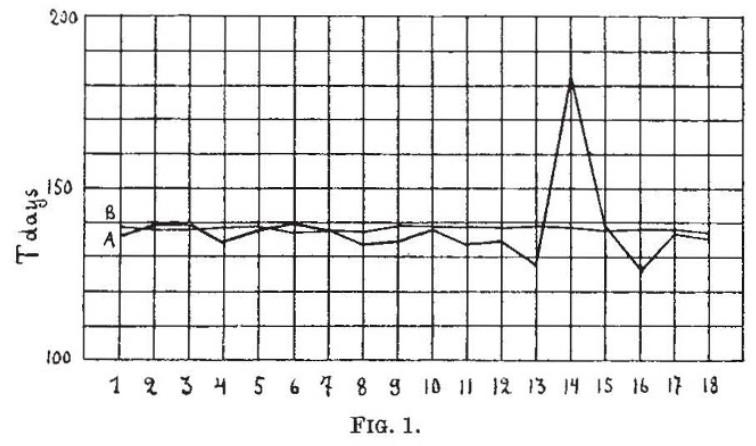

the rapidity of the decay must be influenced by the quantity of radioactive substance which is included in the great bulk of the basic rock. The greater the quantity, the less must be the decay in this place because of the absorption of radiant energy, causing the radioactive decay of heavy atoms, by radioactive elements in the underlying layers.

In favour of this assumption also, we have the fact that the greatest deviations of the period occur in places with disturbed tectonics, that is, in the places situated in Caucasus and the region adjoining it, on its northern boundary.

L. N. Bogojavlensky. Leningrad, April 18.

\section{Thyroid and Temperature in Cold-blooded Vertebrates.}

This problem which Prof. Huxley discusses in NATURE of May 11, p. 712 , is a very intriguing one. To me it appears to have more difficulties than Prof. Huxley allows for. He begins by saying that "it is well known that the thyroid is concerned with temperature regulation in homothermic animals". In my recently published book, "Fever, Heat Regulation, Climate, and the Thyroid Adrenal Apparatus ", I have reviewed the very scanty and contradictory literature on this subject, and one could scarcely say that the relationship of the thyroid gland to heat regulation has been previously either well known or well understood. I dare scarcely hope that my own views on the problem as set forth in a book published only a year ago have already been assimilated so completely as to have become a commonplace of scientific literature.

In the book mentioned I directed attention to the difficulties of the problem discussed by Prof. Huxley. In warm-blooded animals a change of the thermal environment from heat to cold stimulates the thyroid and adrenal glands to increased activity, and there is a rise in general metabolism. Exposure to heat produces the opposite effect: it induces a resting condition in the thyroid and adrenal glands and the metabolism is lowered. The resting condition of the

No. 3110 , VoL. 123] 